\title{
Derivation of Generalized Young's Equation for Wetting of Cylindrical Droplets on Rough Solid Surface
}

\author{
Xiao-Song Wang ${ }^{1}$ \\ ${ }^{1}$ Institute of Mechanical and Power Engineering, Henan Polytechnic University, No. 2001, Century Avenue, \\ Jiaozuo, Henan 454003, China \\ Correspondence: Xiao-Song Wang, Institute of Mechanical and Power Engineering, Henan Polytechnic \\ University, No. 2001, Century Avenue, Jiaozuo, Henan 454003, China. Tel: 86-391-3987543. E-mail: \\ wangxs2016@163.com
}

Received: June 27, 2015 Accepted: July 24, 2015 Online Published: August 7, 2015

doi:10.5539/mer.v5n2p17 URL: http://dx.doi.org/10.5539/mer.v5n2p17

\begin{abstract}
The surface tension depends on the radius of curvature of the liquid-vapor interface. For nano-scale wetting phenomena of cylindrical droplets, we should consider the curvature effects of the surface tension and the line tension. However, previous works have not analyzed the influence of the curvature effects of the surface tension. In this paper, we discuss the influence of the curvature effects of the surface tension on the contact angles based the Kim-Lee-Han-Park equation. The hydrophilic wetting of cylindrical droplets on rough and chemically homogeneous non-deformable substrates were studied by methods of thermodynamics. A generalized Young's equation for wetting of cylindrical droplets on chemically homogeneous and rough non-deformable substrates was derived based on the thermodynamic equilibrium conditions. This equation reduces to the Wenzel equation if we ignore the influence of line tension. For contact angles of cylindrical droplets with sufficiently large radii, a generalized Young's equations were derived considering the curvature effects of the surface tension.
\end{abstract}

Keywords: contact angle, wetting, Young's equation, Wenzel equation, surface tension, curvature effect, cylindrical droplet

\section{Introduction}

Wetting phenomena are common in solid-liquid-gas systems, for instance, wetting of liquid droplets on solid surfaces, adhesives, lubricants and capillary penetration in to porous media (Adamson, 1990; Gennes, Brochard-Wyart, \& Quere, 2004). Wetting abilities are important in many industrial applications, for example, the wetting abilities of electrolytes on electrodes plays a key role in improving the specific energy density of supercapacitors (Kim, Koo, Lee, \& Braun, 2014) and lithium-ion batteries (Pfleging \& Proella, 2014).

In 1805, Thomas Young argued that the contact angle $\theta_{Y}$ for the wetting of spherical droplets on rough and chemically homogeneous substrates is determined by the following equation (Young, 1805)

$$
\cos \theta_{Y}=\frac{\sigma_{S G}-\sigma_{S L}}{\sigma_{L G}}
$$

where $\theta_{Y}$ is the contact angle, $\sigma_{L G}$ is the surface tension of the liquid - vapor interface corresponds to the choice of the surface of tension as a dividing surface, $\sigma_{S G}$ is the surface free energy per unit area of the solid - vapor interface, $\sigma_{S L}$ is the surface free energy per unit area of the solid - liquid interface.

Now, Equation (1.1) is called the Young's equation. The Young's equation Equation (1.1) is widely applied to macroscopic capillary phenomena (Pfleging \& Proella, 2014; Xiao-Song et al., 2014).

In 1878, Gibbs for the first time gave a theoretical derivation of the Young's equation Equation (1.1) based on the theory of thermodynamics (Gibbs, 1928). Since then, many theoretical research works have been carried out (Pfleging \& Proella, 2014).

The surface tension depends on the radius of curvature of the liquid-vapor interface. For nano-scale wetting phenomena of cylindrical droplets, we should consider the curvature effects of the surface tension and the line tension. However, previous works have not analyzed the influence of the curvature effects of the surface tension. 
The purpose of this paper is to present a theoretical study the contact angles of cylindrical droplets on hydrophilic substrates and discuss the influence of the curvature effects of the surface tension on the contact angles.

\section{The Helmholtz Free Energy for Wetting of a Cylindrical Droplet on Rough Substrate}

Consider a single-component cylindrical liquid droplet in contact with chemically homogeneous and rough substrates.

If the contact angle less than $90^{\circ}$, then we say that the solid surface is hydrophilic. If the contact angle larger than $90^{\circ}$, then we say that the solid surface is hydrophobic. We only consider the wetting of a cylindrical droplet on hydrophilic solid surface. An illustration of hydrophilic wetting is shown in Figure 1.

Introducing Gibbs's concept of dividing surface and the concept of dividing line (Gibbs, 1928; Ono \& Kondo, 1960; Rowlinson \& Widom, 1982), the above solid-liquid-vapor system can be divided into six subsystems, i.e. liquid phase, vapor phase, the liquid-vapor interface, the solid-liquid interface, the solid-vapor interface and the three-phase contact line.

Therefore, the total Helmholtz free energy F of the system is the sum of the Helmholtz free energies of these seven parts. Thus, we have

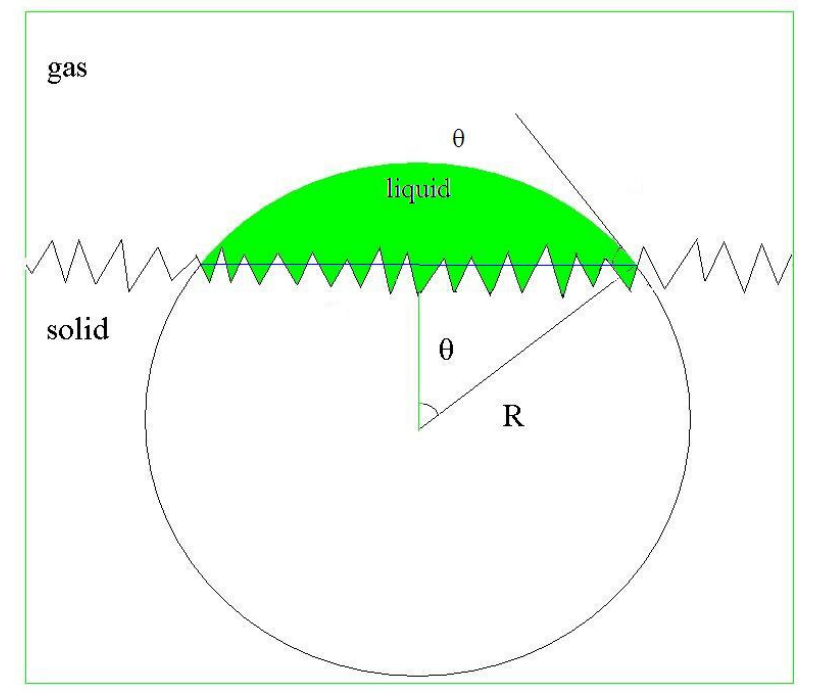

Figure 1 an illustration of hydrophilic wetting of a cylindrical droplet on a rough substrate.

$$
\mathrm{F}=\mathrm{F}_{\mathrm{L}}+\mathrm{F}_{\mathrm{G}}+\mathrm{F}_{\mathrm{LG}}+\mathrm{F}_{\mathrm{SL}}+\mathrm{F}_{\mathrm{SG}}+\mathrm{F}_{\mathrm{SLG}}
$$

where $\mathrm{F}$ is the total Helmholtz free energy, $\mathrm{F}_{\mathrm{L}}, \mathrm{F}_{\mathrm{G}}, \mathrm{F}_{\mathrm{LG}}, \mathrm{F}_{\mathrm{SL}}, \mathrm{F}_{\mathrm{SG}}$ and $\mathrm{F}_{\mathrm{SLG}}$ are the Helmholtz free energies of the seven parts respectively.

The Helmholtz free energies of these seven parts can be written as

$$
\begin{gathered}
\mathrm{F}_{\mathrm{L}}=-\mathrm{p}_{\mathrm{L}} \mathrm{V}_{\mathrm{L}}+\mu_{\mathrm{L}} \mathrm{N}_{\mathrm{L}} \\
\mathrm{F}_{\mathrm{G}}=-\mathrm{p}_{\mathrm{G}} \mathrm{V}_{\mathrm{G}}+\mu_{\mathrm{G}} \mathrm{N}_{\mathrm{G}} \\
\mathrm{F}_{\mathrm{LG}}=\sigma_{\mathrm{LG}} \mathrm{A}_{\mathrm{LG}}+\mu_{\mathrm{LG}} \mathrm{N}_{\mathrm{LG}} \\
\mathrm{F}_{\mathrm{SL}}=\sigma_{\mathrm{SL}} \mathrm{A}_{\mathrm{SL}}+\mu_{\mathrm{SL}} \mathrm{N}_{\mathrm{SL}} \\
\mathrm{F}_{\mathrm{SG}}=\sigma_{\mathrm{SG}} \mathrm{A}_{\mathrm{SG}}+\mu_{\mathrm{SG}} \mathrm{N}_{\mathrm{SG}} \\
\mathrm{F}_{\mathrm{SLG}}=\kappa \mathrm{L}_{\mathrm{SLG}}+\mu_{\mathrm{SLG}} \mathrm{N}_{\mathrm{SLG}}
\end{gathered}
$$

where $p_{L}$ and $p_{G}$ are the pressures of the liquid phase and the vapor phase respectively, $V_{L}$ and $V_{G}$ are the volumes of the liquid phase and the vapor phase respectively, $\mu$ is the chemical potential of the six subsystems, $N_{L}, N_{G}, N_{L G}, N_{S L}, N_{S G}$ and $N_{S L G}$ are the mole numbers of molecules of the liquid phase, the vapor phase, the liquid - vapor interface, the solid - liquid interface, the solid - vapor interface and the three - phase contact line respectively, $A_{L G}, A_{S L}$ and $A_{S G}$ are the surface area of the liquid - vapor interface, the solid - liquid interface, the solid - vapor interface respectively, $\sigma_{L G}, \sigma_{S L}$ and $\sigma_{S G}$ are the surface tensions of the liquid - vapor interface, the 
solid - liquid interface, the solid - vapor interface respectively, $L_{S L G}$ is the value of the length of the three - phase contact line, $k$ is the line tension.

In order to calculate the geometrical quantities in the above equations, we may introduce the following assumption:

Assumption 1: Suppose the equilibrium shape of a droplet on a rough and homogeneous solid substrate is a part of a cylinder which was cut by a plane parallel to the axis of the cylinder.

Based on Assumption 1, the total Helmholtz free energy $\mathrm{F}$ of the system is

$$
\begin{aligned}
\mathrm{F}= & -\left(\mathrm{p}_{\mathrm{L}}-\mathrm{p}_{\mathrm{G}}\right)(\theta-\sin \theta \cos \theta) R^{2} L \\
& -\mathrm{p}_{\mathrm{G}} \mathrm{V}_{\mathrm{t}}+\mu \mathrm{N}_{\mathrm{L}}+\mu \mathrm{N}_{\mathrm{G}} \\
& +\sigma_{\mathrm{LG}} 2 \theta \mathrm{RL}+\mu \mathrm{N}_{S L}+\mu \mathrm{N}_{\mathrm{LG}} \\
& +r_{s}\left(\sigma_{\mathrm{SL}}-\sigma_{\mathrm{SG}}\right) 2 \mathrm{R} \sin \theta L \\
& +r_{s} \sigma_{\mathrm{SG}} \mathrm{A}_{\mathrm{t}}+\mu \mathrm{N}_{\mathrm{SG}} \\
& +2 r_{L} \mathrm{~L} \kappa+\mu \mathrm{N}_{\mathrm{SLG}},
\end{aligned}
$$

where $\mathrm{R}$ is the radius of the cylindrical liquid droplet, $\theta$ is the contact angle, $\mathrm{L}$ is the length of the

cylindrical liquid droplet, $\mathrm{A}_{\mathrm{t}}$ is the total surface area, $r_{s}$ is the surface roughness factor, $r_{L}$ is the line roughness factor.

\section{Generalized Young's Equations based on Thermodynamic Equilibrium Condition}

The purpose of this section is to derive a generalized Young's equations for cylindrical droplets on rough and homogeneous solids by methods of thermodynamics.

According to Gibbs's concept of dividing surface (Ono \& Kondo, 1960), we can choose an arbitrary conformal surface as a dividing surface. Now, we suppose that the radius $\mathrm{R}$ of the dividing surface has already been chosen according to some fixed conditions. The contact angle $\theta$ now becomes variable. The thermodynamic equilibrium condition at a fixed temperature $\mathrm{T}$ of an open system is (Nijmeijer, Bruin, Woerkom, \& Bakker, 1992)

$$
\left(\frac{\partial F}{\partial \theta}\right)_{T, \mu}=0
$$

where the subscript $T$ and $\mu$ stands for fixed temperature $T$ and fixed chemical potential $\mu$.

It is convenient to introduce the concept of grand potential to treat an open system. The definition of the grand potential $\Omega$ of a system is (Rowlinson \& Widom, 1982)

$$
\Omega=\sum_{\mathrm{i}=1}^{\tau}\left(\mathrm{F}_{\mathrm{i}}-\mu_{\mathrm{i}} \mathrm{N}_{\mathrm{i}}\right)
$$

where $\tau$ is the number of subsystems of the system, $\mathrm{F}_{\mathrm{i}}$ is the Helmholtz free energy of the $\mathrm{i}-$ th subsystem, $\mu_{\mathrm{i}}$ is the chemical potential of the the $\mathrm{i}$-th subsystem, $\mathrm{N}_{\mathrm{i}}$ is the mole numbers of molecule of the the $\mathrm{i}$-th subsystem. Putting Equation (3.2) into Equation (3.1), the thermodynamic equilibrium condition becomes (Nijmeijer, Bruin, Woerkom, \& Bakker, 1992)

$$
\left(\frac{\partial \Omega}{\partial \theta}\right)_{T, \mu}=0
$$

Putting Equation (2.8) into Equation (3.2), the total grand potential $\Omega$ of the above system is

$$
\begin{aligned}
\Omega= & -\left(\mathrm{p}_{\mathrm{L}}-\mathrm{p}_{\mathrm{G}}\right)(\theta-\sin \theta \cos \theta) R^{2} L \\
& -\mathrm{p}_{\mathrm{G}} \mathrm{V}_{\mathrm{t}}+\sigma_{\mathrm{LG}} 2 \theta \mathrm{RL} \\
& +r_{s}\left(\sigma_{\mathrm{SL}}-\sigma_{\mathrm{SG}}\right) 2 \mathrm{R} \sin \theta L \\
& +r_{s} \sigma_{\mathrm{SG}} \mathrm{A}_{\mathrm{t}}+2 r_{L} \mathrm{~L} \kappa .
\end{aligned}
$$

Putting Equation (3.4) into Equation (3.3), we have 


$$
\begin{aligned}
& -\left(\mathrm{p}_{\mathrm{L}}-\mathrm{p}_{\mathrm{G}}\right)\left(\frac{\partial f_{1}}{\partial \theta}\right)_{T, \mu}-f_{1}\left(\frac{\partial\left(\mathrm{p}_{\mathrm{L}}-\mathrm{p}_{\mathrm{G}}\right)}{\partial \theta}\right)_{T, \mu} \\
& +\sigma_{\mathrm{LG}}\left(\frac{\partial f_{2}}{\partial \theta}\right)_{T, \mu}+f_{2}\left(\frac{\partial \sigma_{\mathrm{LG}}}{\partial \theta}\right)_{T, \mu} \\
& +r_{s}\left(\sigma_{\mathrm{SG}}-\sigma_{\mathrm{SL}}\right)\left(\frac{\partial f_{3}}{\partial \theta}\right)_{T, \mu}+f_{3} r_{s}\left(\frac{\partial\left(\sigma_{\mathrm{SG}}-\sigma_{\mathrm{SL}}\right)}{\partial \theta}\right)_{T, \mu} \\
& +r_{s} \sigma_{\mathrm{SG}}\left(\frac{\partial f_{4}}{\partial \theta}\right)_{T, \mu}+f_{4} r_{s}\left(\frac{\partial \sigma_{\mathrm{SG}}}{\partial \theta}\right)_{T, \mu} \\
& +r_{L} \kappa\left(\frac{\partial f_{5}}{\partial \theta}\right)_{T, \mu}+f_{5} r_{L}\left(\frac{\partial \kappa}{\partial \theta}\right)_{T, \mu}=0,
\end{aligned}
$$

where

$$
\begin{gathered}
f_{1}=(\theta-\sin \theta \cos \theta) R^{2} L, \\
f_{2}=2 \theta R L, \\
f_{3}=2 L R \sin \theta, \\
f_{4}=\mathrm{A}_{\mathrm{t},} \\
f_{5}=2 L .
\end{gathered}
$$

According to Gibbs's concept of dividing surface (Gibbs, 1961; Rowlinson \& Widom, 1982), we can choose an arbitrary conformal surface as a dividing surface. Now, the radius $\mathrm{R}$ of the dividing surface has already been chosen according to some fixed conditions. At the fixed temperature $\mathrm{T}$ and fixed chemical potential, the pressure $\mathrm{p}_{\mathrm{L}}, \mathrm{p}_{G}$ and surface tension will not influence the contact angle $\theta$. Thus, in order to simplify Equation (3.5), we introduce the following assumption.

Assumption 2: Suppose the following equations are valid for the wetting of cylindrical droplets on rough and chemically homogeneous non - deformable substrates

$$
\begin{gathered}
\left(\frac{\partial\left(\mathrm{p}_{\mathrm{L}}-\mathrm{p}_{G}\right)}{\partial \theta}\right)_{T, \mu}=0, \\
\left(\frac{\partial \sigma_{S L}}{\partial \theta}\right)_{T, \mu}=0, \\
\left(\frac{\partial \sigma_{\mathrm{SG}}}{\partial \theta}\right)_{T, \mu}=0, \\
\left(\frac{\partial \sigma_{\mathrm{LG}}}{\partial \theta}\right)_{T, \mu}=0 .
\end{gathered}
$$

We have the following results

$$
\begin{gathered}
\left(\frac{\partial f_{1}}{\partial \theta}\right)_{T, \mu}=2 R^{2} L \sin ^{2} \theta, \\
\left(\frac{\partial f_{2}}{\partial \theta}\right)_{T, \mu}=2 R L, \\
\left(\frac{\partial f_{3}}{\partial \theta}\right)_{T, \mu}=2 R L \cos \theta, \\
\left(\frac{\partial f_{4}}{\partial \theta}\right)_{T, \mu}=0, \\
\left(\frac{\partial f_{5}}{\partial \theta}\right)_{T, \mu}=0,
\end{gathered}
$$


Putting Equations (3.11-3.19) into Equation (3.5), we obtain

$$
\text { - }\left(\mathrm{p}_{\mathrm{L}}-\mathrm{p}_{\mathrm{G}}\right) R \sin ^{2} \theta+\sigma_{\mathrm{LG}}+r_{s}\left(\sigma_{S L}-\sigma_{S G}\right) \cos \theta+\frac{r_{L}}{R}\left(\frac{\partial \kappa}{\partial \theta}\right)_{T, \mu i}=0 .
$$

It is known that a generalized Laplace's equation of a free cylindrical droplet in vapor can be written as (Wenzel, 1936)

$$
p_{L}-p_{G}=\frac{\sigma_{L G}}{R}+\left[\frac{d \sigma_{L G}}{d R}\right]
$$

Where the differential in square bracket[ ] denotes the change resulted from a mathematical variation of the position of this dividing surface by the amount $d R$ in the same physical system under the same fixed physical state. Applying Equation (3.21), Equation (3.20) becomes

$$
\begin{aligned}
\cos \theta & =r_{s} \frac{\sigma_{S G}-\sigma_{S L}}{\sigma_{L G}}+\frac{R \sin \theta \tan \theta}{\sigma_{\mathrm{LG}}}\left[\frac{\mathrm{d} \sigma_{\mathrm{LG}}}{\mathrm{dR}}\right] \\
& -\frac{r_{L}}{\sigma_{\mathrm{LG}} \mathrm{R} \cos \theta}\left(\frac{\partial \kappa}{\partial \theta}\right)_{T, \mu i} .
\end{aligned}
$$

Equation (3.22) is a generalized Young's equation for wetting of a cylindrical droplet on chemically homogeneous and rough non - deformable substrates. Equation (43) is the main results of this work.

Following Gibbs (Kim, Lee, Han, \& Park, 2006; Nijmeijer, Bruin, Woerkom, \& Bakker, 1992), we introduce the concept of surface of tension $\mathrm{M}_{s}$ as follows

$$
\left[\left.\frac{\mathrm{d} \sigma_{\mathrm{LG}}}{\mathrm{dR}}\right|_{R=R_{s}}=0\right.
$$

Where $\mathrm{R}_{s}$ is the radius of the surface of tension $M_{s}$

If we choose the surface of tension $M_{s}$ as the dividing surface, then Equation (43) becomes

$$
\cos \theta=r_{s} \frac{\sigma_{S G}-\sigma_{S L}}{\sigma_{L G}}-\frac{r_{L}}{\sigma_{\mathrm{LG}} \mathrm{R} \cos \theta}\left(\frac{\partial \kappa}{\partial \theta}\right)_{T, \mu i} .
$$

Equation (3.24) is a useful generalization of the Young's equations Equation (1.1) for wetting of cylindrical droplets.

If we neglect the second term on the right side of Equation (3.24), we have

$$
\cos \theta=r_{s} \frac{\sigma_{S G}-\sigma_{S L}}{\sigma_{L G}} .
$$

Equation (3.25) is the Wenzel equation (Wenzel, 1936).

\section{Brief Review of Curvature Effects of Surface Tension of Cylindrical Droplet}

In 2006, Kim, Lee, Han, and Park (2006) obtained the following equation for the curvature effects of surface tension of cylindrical droplets

$$
\frac{1}{\sigma} \frac{d \sigma_{L G}\left(R_{s}\right)}{d R_{s}}=\frac{\frac{\delta_{G}}{R_{s}^{2}}\left(1+\frac{\delta_{G}}{2 R_{s}}\right)}{1+\frac{\delta_{G}}{R_{s}}+\frac{\delta_{G}^{2}}{2 R_{s}^{2}}},
$$

where $\sigma_{L G}\left(R_{s}\right)$ is the surface tension of a cylindrical droplet with radius $R_{s}, R_{s}$ is the radius of Gibbs' surface of tension, $\delta_{G} \equiv R_{e}-R_{s}, R_{e}$ is the radius corresponding to the equimolar dividing surface.

For sufficiently large droplets, we neglect $\delta_{G}^{3} / R_{s}^{3}$ and $\delta_{G}^{2} / R_{s}^{2}$, and treat $\delta_{G}$ as the constant $\delta_{T}$, Equation (4.1) can be solved. The result is

$$
\frac{\sigma_{L G}\left(R_{s}\right)}{\sigma_{\infty}}=\exp \left(-\frac{\delta_{T}}{R_{s}}\right),
$$


where $\sigma_{\infty}$ is the surface tension of plane surface with radius $R_{s}=\infty, \delta_{T} \equiv \lim _{R \rightarrow \infty} \delta_{G}$. $\delta_{T}$ is usually called the Tolman length in the literatures (Rowlinson \& Widom, 1982).

We have the following Taylor's expansion

$$
\exp (-x)=1-x+\frac{x^{2}}{2 !}-\frac{x^{3}}{3 !}+\ldots
$$

Applying Equation (4.3), Equation (4.2) can be expanded as

$$
\frac{\sigma_{L G}\left(R_{s}\right)}{\sigma_{\infty}}=1-\frac{\delta_{T}}{R_{s}}+\frac{\delta_{T}^{2}}{R_{s}^{2}}+\ldots
$$

\section{Generalized Young's Equation Considering the Curvature Effects of the Surface Tension}

Using Equation (4.2), Equation (3.24) can be written as

$$
\sigma_{\infty} \exp \left(-\frac{\delta_{T}}{R_{s}}\right) \cos \theta=r_{s}\left(\sigma_{S G}-\sigma_{S L}\right)-\frac{r_{L}}{\mathrm{R} \cos \theta}\left(\frac{\partial \kappa}{\partial \theta}\right)_{T, \mu} .
$$

Equation (5.1) is a generalized Young's equation for spherical droplets on rough but chemically heterogeneous non-deformable substrates considering the curvature effects of the surface tension

$\sigma_{L G}$. Equation (5.1) is one of the main results of this work.

Applying Equation (4.3), Equation (5.1) becomes

$$
\sigma_{\infty}\left(1-\frac{\delta_{T}}{R_{s}}+\frac{\delta_{T}^{2}}{R_{s}^{2}}+\ldots\right) \cos \theta=r_{s}\left(\sigma_{S G}-\sigma_{S L}\right)-\frac{r_{L}}{\mathrm{R} \cos \theta}\left(\frac{\partial \kappa}{\partial \theta}\right)_{T, \mu} .
$$

According to experiments (Adamson, 1990), the curvature effects of the surface tension $\sigma_{L G}$ of macroscopic cylindrical droplets can be neglected. However, for nano-scale cylindrical droplets, the curvature effects of the surface tension $\sigma_{L G}$ are important (Wenzel, 1936; Rowlinson \& Widom, 1982; Adamson, 1990; Nijmeijer, Bruin, Woerkom, \& Bakker, 1992; Gennes, Brochard-Wyart, \& Quere, 2004;). For droplets with sufficiently large radii, if we neglect $\frac{\delta_{T}^{2}}{R_{s}^{2}}$ and higher terms in Equation (5.2), then we have

$$
\sigma_{\infty}\left(1-\frac{\delta_{T}}{R_{s}}\right) \cos \theta=r_{s}\left(\sigma_{S G}-\sigma_{S L}\right)-\frac{r_{L}}{\mathrm{R} \cos \theta}\left(\frac{\partial \kappa}{\partial \theta}\right)_{T, \mu} .
$$

\section{Conclusion}

The hydrophilic wetting of cylindrical droplets on rough and chemically homogeneous non-deformable substrates were studied by methods of thermodynamics. A generalized Young's equation for wetting of cylindrical droplets on chemically homogeneous and rough non-deformable substrates was derived based on the thermodynamic equilibrium conditions. This equation reduces to the Wenzel equation if we ignore the influence of line tension. It is known that the surface tension depend on the radius of curvature of the liquid-vapor interface. For nano-scale wetting phenomena, we should consider the curvature effects of the surface tension and the line tension. However, previous works have not analyzed the influence of the curvature effects of the surface tension. Based the Kim-Lee-Han-Park equation, we discuss the influence of the curvature effects of the surface tension on the contact angles. For contact angles of cylindrical droplets with sufficiently large radii, a generalized Young's equations were derived considering the curvature effects of the surface tension.

\section{Acknowledgments}

This work was supported by the Key Project of Science of the Education Bureau of Henan Province (Grant No. 15A130001) and the Doctor Research Foundation of Henan Polytechnic University (Grant No. 72515 - 466).

\section{References}

Adamson, A. W. (1990). Physical Chemistry of Surfaces. New York: John Wiley Sons.

Gennes, P. G. d., Brochard-Wyart, F., \& Quere, D. (2004). Capillarity and wetting phenomena: drops, bubbles, pearls waves. New York: Springer-Verlag. 
Gibbs, J. W. (1928). The Collected Works of J. Willard Gibbs (vol. 1, Thermodynamics). New Haven: Yale Univ. Press.

Gibbs, J. W. (1961). The Scientific Papers of J. W. Gibbs (Vol. 1). New York: Dover.

Kim, B. G., Lee, J. S., Han, M., \& Park, S. (2006). A molecular dynamics study on stability and thermophysical properties of nanoscale liquid threads. Nanoscale and Microscale Thermophysical Engineering 10, 283. http://dx.doi.org/10.1080/15567260600902061

Kim, S. K., Koo, H. J., Lee, A., \& Braun, P. V. (2014). Selective Wetting-Induced Micro-Electrode Patterning for Flexible Micro-Supercapacitors. Advanced Materials, 26, 5108. http://dx.doi.org/10.1002/adma.201401525

Nijmeijer, M. J. P., Bruin, C., Woerkom, A. B. V., \& Bakker, A. F. (1992). Molecular dynamics of the surface tension of a drop. J. Phys. Chem., 96, 565. http://dx.doi.org/10.1063/1.462495

Ono, S., \& Kondo, S. (1960). Molecular Theory of Surface Tension in Liquids. In S. Flugge (Ed.), Encyclopedia of Physics (volume 10). Berlin: Springer-Verlag.

Pfleging, W., \& Proella, J. (2014). A new approach for rapid electrolyte wetting in tape cast electrodes for lithium-ion batteries. Journal of Materials Chemistry A, 2, 14918. http://dx.doi.org/10.1039/C4TA02353F

Rowlinson, J. S., \& Widom, B. (1982). Molecular Theory of Capillarity. Oxford: Clarendon Press.

Wenzel, R. N. (1936). Resistance of solid surfaces to wetting by water. Industrial \& Engineering Chemistry, 28(8), 988-994.

Xiao-Song, W., Shu-Wen, C., Long, Z., Sheng-Hua, X., Zhi-Wei, S., \& Ru-Zeng, Z. (2014). A Generalized Young's Equation for Contact Angles of Droplets on Homogeneous and Rough Substrates. J. Adhesion Sci. Tech., 28, 161. http://dx.doi.org/10.1080/01694243.2013.833401

Young, T. (1805). An essay on the cohesion of fluids. Philos. Trans. Roy. Soc. London, 65.

\section{Copyrights}

Copyright for this article is retained by the author(s), with first publication rights granted to the journal.

This is an open-access article distributed under the terms and conditions of the Creative Commons Attribution license (http://creativecommons.org/licenses/by/3.0/). 